눈 개회충증 환자에서의 혈청 안지오텐신저환효소 수치 상승

\title{
Elevation of Serum Angiotensin-Converting Enzyme Level in Patients with Ocular Toxocariasis
}

\author{
정형기, 이정현, 황덕진 \\ Hyeong Gi Jung, Jung Hyun Lee, Duck Jin Hwang \\ 한길안과병원 \\ HanGil Eye Hospital, Incheon, Korea
}

Purpose: Serum angiotensin-converting enzyme (ACE) level is regarded as an important disease marker, particularly for sarcoidosis. The purpose of this study was to assess whether high serum ACE levels are present in uveitic patients with ocular toxocariasis.

Case summary: Six patients newly diagnosed with ocular toxocariasis within the last three months were investigated. Toxocariasis infection was confirmed by serum laboratory tests in all patients and by additional vitreous sampling in three patients. Three patients showed elevated serum ACE levels. None of these patients suffered any known systemic disease or showed evidence of sarcoidosis as assessed by chest high-resolution computed tomography.

Conclusions: Serum ACE level may be elevated in patients with ocular toxocariasis and may serve as a marker of toxocariasis.

Keywords: Angiotensin converting enzyme; Toxocariasis

\section{Introduction}

Angiotensin-converting enzyme (ACE) is mainly expressed in pulmonary vessel endothelium, where it converts angiotensin I to angiotensin II, resulting in vasoconstriction [1,2]. Lieberman first reported elevated serum ACE levels in sarcoidosis [3]. Furthermore, serum ACE levels may be elevated in many diseases, including Gaucher's disease, leprosy, lymphoma, diabetes, hyperthyroidism, liver cirrhosis, and pulmonary tuberculosis [4]. Recently, several studies reported an association between ACE gene polymorphisms and rheumatologic disease, including ankylosing spondylitis and Behcet's disease [5,6].

We herein report three cases of confirmed uveitic toxocariasis infection, which presented with elevated serum ACE levels. These patients had no evidence of sarcoidosis or any of the other above-mentioned systemic diseases.

\section{Case Report}

Six patients newly diagnosed with ocular toxocariasis during

\section{Address reprint requests to Duck Jin Hwang, MD}

Department of Ophthalmology, HanGil Eye Hospital, \#35 Bupyeong-daero, Bupyeong-gu, Incheon 21388, Korea Tel: 82-32-503-3322, Fax: 82-32-504-3322

E-mail:hallelu7@gmail.com
Received: 2016. 4. 22

Revised: 2016. 4. 30

Accepted: 2016. 5. 2 
the past three months were investigated. All patients had confirmed toxocariasis infection, as assessed by serum laboratory tests. Additionally, infection was confirmed by vitreous sampling in three of these patients. Three out of six patients with confirmed ocular toxocariasis showed elevated serum ACE levels.

\section{Case 1}

A 53-year-old woman presented with decreased vision in her right eye. She had no pertinent medical history. At the first visit, visual acuity in her right eye was 20/40. Trace inflammatory cells in the anterior chamber and the anterior vitreous cavity, as well as mild vitreous opacity, were found on slit lamp examination. On fundus examination, a granulomatous mass-like lesion was found near the macula (Fig. 1A). Fluorescein angiography (FAG) revealed dye leakage from the mass-like lesion (Fig. 2A). Laboratory tests were positive for toxocara $\mathrm{IgG}$ antibody and serum ACE level was elevated (79.3 U/L). Tests for toxoplasma, syphilis, and human immunodeficiency virus (HIV) all yielded negative results. The patient was referred to an internist for evaluation of systemic disease. No evidence of systemic disease, including sarcoidosis or tuberculosis, was ascertained. High-resolution computed tomography (HRCT) did not show any abnormalities. We prescribed oral prednisolone (starting dose $40 \mathrm{mg}$ q.d. and tapered during several weeks) and oral albendazole (800 mg q.d. for 3 weeks). The vitreous opacity and inflam- matory cells disappeared with treatment, and visual acuity recovered to $20 / 30$.

\section{Case 2}

A 48-year-old woman presented with decreased vision in her right eye. She had a medical history of uveitis of unknown etiology. At the initial visit, visual acuity was $20 / 100$. On slit lamp examination, inflammatory cells were detected both in the anterior chamber and the anterior vitreous, and vitreous opacities were present (Fig. 1B). A granulomatous mass-like lesion was found on fundus examination, located inferotemporally to the macula. FAG showed dye staining near the mass and retinal vessels (Fig. 2B). Laboratory results were identical to those presented for Case 1 above, i.e., positive for toxocara antibody and high serum ACE level (68.6 U/L). Laboratory results for toxoplasma, syphilis, and HIV were all negative. We also referred the patient to an internist, but there was no evidence of systemic disease, including sarcoidosis or tuberculosis. Chest HRCT findings were normal. We prescribed oral prednisolone and albendazole as above, however, vitreous opacities gradually worsened. Cataract surgery (phacoemulsification and posterior chamber lens [PCL] implantation), vitrectomy, and vitreous sampling were performed. The collected vitreous was positive for anti-toxocara antibody. After surgery, inflammation subsided and visual acuity improved to 20/50.
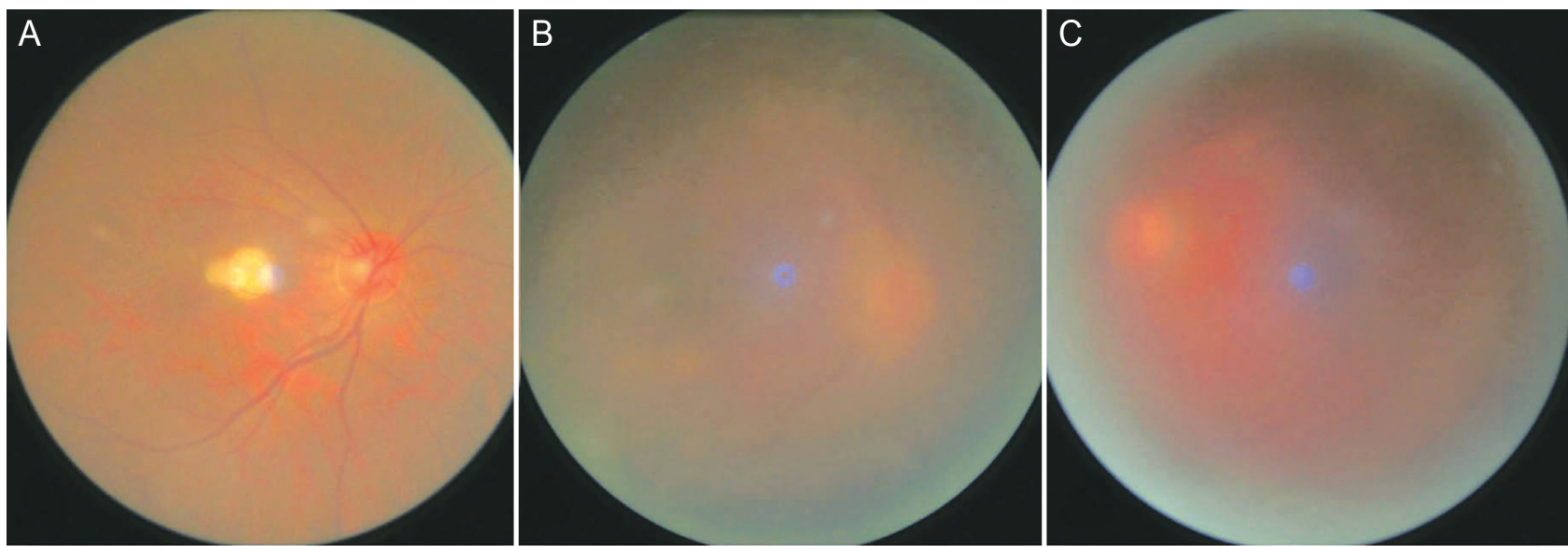

Figure 1. Fundus photos of the patients for each case. (A) Case 1. Fundus image showing a round granulomatous, exudative lesion near the fovea. (B) Case 2. Vitreous opacities with an exudative granulomatous lesion which is faintly visible found in the patient's right eye. (C) Case 3. Vitreous opacities found in the patient's left eye. 

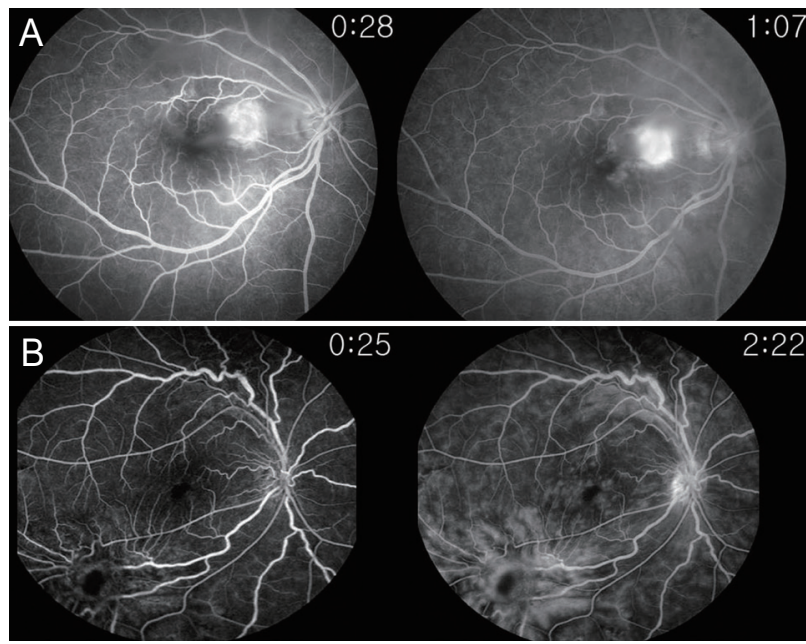

Figure 2. Fluorescein angiogarphy (FAG) of the patients in case 1 and 2. (A) Case 1. FAG of the patinet that revealed dye leakage at both early and late phase. (B) Case 2. FAG of the patient showed dye stain near the mass-like lesion and retinal vessels.

\section{Case 3}

A 53-year-old man was referred to us from a local clinic for uncontrolled uveitis of unknown etiology present for two months. Visual acuity of the left eye was $20 / 200$. Significant amounts of inflammatory cells were found in the anterior chamber and the anterior vitreous, and vitreous opacity was present (Fig. 1C). On fundus examination, an exudative mass-like lesion was found. Laboratory test results were similar to those described above, i.e., positive for anti-toxocara antibody and showing elevated serum ACE level (67.7 U/L). Rheumatologic markers, including rheumatoid factor, anti-nuclear antibody, anti-neutrophil cytoplasmic antibody, human leukocyte antigen-B27, and other microorganism markers, including cytomegalovirus, herpes simplex virus, Varicella-Zoster virus, toxoplasma, syphilis, and HIV, were all negative. We referred the patient to an internist, who did not find evidence of any systemic disease, including sarcoidosis or tuberculosis. Similarly, chest HRCT did not suggest the presence of systemic disease. The patient requested early intervention due to prolonged uncontrolled inflammation and his desire to return back to work. We performed cataract surgery, consisting of phacoemulsification and PCL implantation, vitrectomy, and vitreous sampling. The collected vitreous was positive for anti-toxocara antibody. After surgery, inflammation subsided significantly and visual acuity improved to $20 / 60$.

\section{Discussion}

ACE is an 837-amino-acid-long molecule with $129 \mathrm{kDa}$ in weight. It is expressed in the endothelium of pulmonary capillaries, liver, adrenal glands, pancreas, spleen, and heart $[1,7,8]$. $\mathrm{ACE}$ is a regulatory enzyme of the renin-angiotensin system, which plays an important role in the control of inflammatory processes through stimulation of cytokine release.

Elevated serum ACE level in some patients with granulomatous uveitis strongly indicates sarcoid uveitis, even if there are no clinical, radiologic or histologic findings suggestive of systemic sarcoidosis [9]. There are several theories that attempt to explain the reason for elevated serum ACE levels in sarcoidosis. For example, it has been suggested that sarcoid cells release a bradykinin-like agent, which stimulates the production of ACE in pulmonary endothelium. Similarly, granuloma and capillary endothelium are thought to produce more ACE during sarcoidosis [3]. Local inflammation as encountered in uveitis could compromise the blood-eye barrier, triggering an exaggerated cellular immune response. Williams et al. studied the epithelioid cells in the granulomas of sarcoidosis and concluded that these cells probably served a synthesizing function [10].

The three cases with elevated serum ACE levels presented herein share some clinical characteristics: first, all were diagnosed with ocular toxocariasis, and all other etiologies for uveitis were ruled out. Second, all patients presented with a granulomatous mass-like lesion in the retina. Importantly, it has previously been reported that some granulomatous diseases, like sarcoidosis, leprosy, and tuberculosis, can lead to elevated serum ACE levels. Last, none of the patients showed any evidence of an underlying systemic disease. Thus, we suggest that serum ACE levels may be elevated in toxocariasis, although its pathophysiology is not yet completely understood.

In summary, we here report three cases of ocular toxocariasis with significantly elevated serum ACE levels. Serum ACE levels may be elevated in patients with ocular toxocariasis and represent a potential biomarker for toxocariasis.

\section{Conflicts of Interest}

The authors report no conflicts of interest. The authors alone are responsible for the content and writing of the paper. 


\section{References}

1. Caldwell PR, Seegal BC, Hsu KC, et al. Angiotensin-converting enzyme: vascular endothelial localization. Science 1976;191:1050-1.

2. Soffer RL. Angiotensin-converting enzyme and the regulation of vasoactive peptides. Annu Rev Biochem 1976;45:73-94.

3. Lieberman J. Elevation of serum angiotensin-converting-enzyme (ACE) level in sarcoidosis. Am J Med 1975;59:365-72.

4. Baughman RP, Lower EE. Sarcoidosis. In: Longo DL, Fauci AS, Kasper DL, et al. eds. Harrison's Principles of Internal Medicine, 18th ed. New York: McGraw-Hill Companies Inc., 2012;v. 2. chap. 329.

5. Inanır A, Yigit S, Tural S, et al. Significant association between insertion/deletion polymorphism of the angiotensin-convertig enzyme gene and ankylosing spondylitis. Mol Vis 2012;18:2107-13.

6. Yigit S, Tural S, Rüstemoglu A, et al. DD genotype of ACE gene I/ $D$ polymorphism is associated with Behcet disease in a Turkish population. Mol Biol Rep 2013;40:365-8.

7. Ryan JW, Ryan US, Schultz DR, et al. Subcellular localization of pulmonary antiotensin-converting enzyme (kininase II). Biochem J 1975;146:497-9.

8. Heinemann HO, Ryan JW, Ryan US. Is the lung a para-endocrine organ? Am J Med 1977;63:595-603.

9. Weinreb RN, Kimura SJ. Uveitis associated with sarcoidosis and angiotensin converting enzyme. Am J Ophthalmol 1980;89:1805.

10. Williams WJ, Erasmus D, James EM, Williams D. The histochemistry and fine structure of epitheloid cells in sarcoidosis. Thorax 1970;25:255 